UCRL-JC-124528

PREPRINT

\title{
Thermal Recovery of NIF Amplifiers
}

\author{
S. Sutton, C. Marshall, C. Petty, \\ L. Smith, B. van Wonterghem, \\ and S. Mills
}

This paper was prepared for submittal to the 2nd Annual International Conference on Solid-State Lasers for Application to Inertial Confinement Fusion Paris, France

October 22-25, 1996

February 1997

This is a preprint of a paper intended for publication in a journal or proceedings. Since changes may be made before publication, this preprint is made available with the understanding that it will not be cited or reproduced without the permission of the author. 


\section{DISCLAIMER}

This document was prepared as an account of work sponsored by an agency of the United States Government. Neither the United States Government nor the University of California nor any of their employees, makes any warranty, express or implied, or assumes any legal liability or responsibility for the accuracy, completeness, or usefulness of any information, apparatus, product, or process

disclosed, or represents that its use would not infringe privately owned rights. Reference herein to any specific commercial product, process, or service by trade name, trademark, manufacturer, or otherwise, does not necessarily constitute or imply its endorsement, recommendation, or favoring by the United States Government or the University of California. The views and opinions of authors expressed herein do not necessarily state or reflect those of the United States Government or the University of California, and shall not be used for advertising or product endorsement purposes. 


\author{
S. Sutton, C. Marshall, C. Petty, L. Smith, \\ B. van Wonterghem, and S. Mills \\ Lawrence Livermore National Laboratory \\ Livermore, California 94550
}

\begin{abstract}
The issue of thermal recovery of the NIF amplifiers has taken on increased emphasis as program goals move toward increasing the shot rate to once every four hours. This paper addresses the technical issues associated with achieving thermal recovery in the NIF amplifiers. We identify two temperature related thermal recovery quantities: (1) the difference between the average slab temperature and the temperature of other surfaces in the amplifier cavity, and (2) the temperature difference in the slab over the aperture. The first quantity relates to optical disturbances in the gas columns in the system, while the second quantity is associated with optical aberations in the laser media itself. Calculations and experiments are used to quantify recovery criteria, and develop cooling approaches. The cooling approaches discussed are (1) active cooling of the flashlamps with ambient gas and chilled gas, and (2) active cooling of the slab edge cladding. Calculations indicate that the NIF baseline cooling approach of $20 \mathrm{cfm}$ per lamp ambient temperature gas flow in both the central and side flashlamp cassettes is capable of meeting thermal recovery requirements for an $\mathbf{8 h o u r}$ shot period, while to achieve a $\mathbf{4}$ hour shot period requires use of chilled gas and edge cladding cooling. In addition, the effect of changing the amplifier cavity and beamtube fill gas from nitrogen to helium is addressed, showing that a factor of $\mathbf{8}$ reduction in the sensitivity to thermal disturbances is possible.
\end{abstract}

Keywords: National Ignition Facility, laser amplifiers, thermal distortions, gas cooling, thermal recovery, numerical simulations

\title{
1. INTRODUCTION
}

The National Ignition Facility (NIF) consists of 192 discrete apertures clustered into 2 wide $x 4$ high bundles. Each 4high aperture stack is separated by a flashlamp cassette creating isolated 1 wide $\mathrm{x} 4$ high cavities confined by sets of Brewster angle slabs. The beamtubes, which are adjacent to the amplifier cavities, are also 4 apertures high. Of particular importance in the design of NIF is development of cooling techniques to remove the residual heat deposited in the slabs, flashlamps, and blastshields in such a manner that optical distortions prior to the next NIF shot are acceptable. The issue of thermal recovery of the NIF amplifiers has taken on increased emphasis as program goals move toward doubling the shot rate from the NIF baseline of once every eight hours, which requires thermal recovery in 7 hours if 1 hour is allotted for realignment, to once every four hours which requires a 3 hour thermal recovery. This has necessitated a renewed look at NIF thermal recovery requirements and the development of technologies to efficiently and economically meet those recovery needs.

We separate thermally related optical distortions in the amplifier and beamtube regions into two distinct categories: (1) optical distortions in the slabs due to temperature gradients across the slab aperture, and (2) optical distortions in the gas columns in the amplifier cavities and beamtubes due to surface temperature difference driven convective flows. Current NIF requirements specify that the residual thermal gradients in the slabs, left-over from the previous shot, shall not contribute greater than 0.04 waves/slab/pass of optical distortion, and that thermal distortions in the gas columns in the amplifier cavities and beamtubes at shot time shall not contribute more than an additional $2.5 \mu \mathrm{rad}$ half-angle beam divergence.

Both requirements place restrictions on temperature non-uniformities allowed in the system, and thus necessitate the development of cooling approaches that can meet or exceed those limits. In the remaining sections of this paper we develop thermal recovery temperature limits associated with the residual optical distortion requirements, and describe cooling techniques that meet the current NIF thermal recovery requirements. To accomplish this task, both numerical simulations and experiments have been used to relate the NIF optical distortion criteria to easily measured and quantified temperature differences in the system. 


\section{*}

Calculations, the details of which follow in the remaining sections of this paper, varied in complexity from a global lumped-mass thermal approximation of the amplifier system to detailed three-dimensional thermal/stress/optic simulations. The lumped-mass model was used to understand the sensitivity of NIF amplifier themal recovery to flashlamp cooling flow characteristics such as gas flowrate and gas temperature. The detailed three-dimensional thermal/stress/optic simulations were used to quantify the effect of temperature gradients across the aperture on optical distortions in the laser slabs.

Experiments employed the Beamlet facility as a vehicle to develop preliminary correlation's between optical distortions and system temperature differences. The Beamlet laser is a 2 wide $\times 2$ high cluster of optical apertures, with only one active aperture. The elements in the remaining three apertures are "dummy" slabs designed to simulate the optical depth of the active laser slabs for radiation pump dynamics purposes. In these experiments, an inactive side module (see Fig. 1a) of the cavity amplifier was modified to allow the measurement of temperatures simultaneously with measurement of optical distortions on the active side aperture. Modifications, depicted in Fig. 1b, consisted of placing a thermocouple instrumented laser slab in this module, in addition to instrumenting the blastshield, architectural glass assembly below the slab, and the top and bottom reflectors. In total, over 30 thermocouples were placed in the amplifier module.

\section{SLAB OPTICAL DISTORTIONS}

Optical distortions in the slabs are directly related to the initial energy distribution in the slab and the cooling mechanisms that are used to remove that energy prior to the next laser shot. Within the slab, temperature gradients are established because the prompt temperature rise in the edge cladding is significantly greater than in the optical aperture. The average prompt temperature rise over the slab aperture is observed to be within the range of $0.5-0.7^{\circ} \mathrm{C}$, while the edge cladding temperature rise is an average of $3^{\circ} \mathrm{C}$. The prompt heating of the flashlamps is estimated as $19^{\circ} \mathrm{C}$ and that of the blastshields measured at roughly $2^{\circ} \mathrm{C}$. Note that for the edge cladding there is approximately a factor of two difference in the temperature rise between the side and top/bottom regions. In the calculations reported in this study, this asymmetry is not included, with the average edge cladding temperature rise used for all edge cladding regions.

The primary heat exchange mechanism in the amplifier cavity is radiation heat transfer between the slabs and blastshields. In the flashlamp cassettes energy is exchanged radiatively between surfaces, but is dominated by convective cooling from the gas flow. During the early stages of thermal recovery, there will be radiant energy exchange from the hotter flashlamps to the colder blastshields and in turn to the slabs. Thus, until the flashlamp cassette cooling flow has significantly reduced the flashlamp temperature there will be a heating of the slabs. Once the flashlamps have been cooled, the process reverses with the energy flow from the slabs to the cooled blastshields.

The edge claddings, on the other hand, are shielded from direct radiant energy exchange to the blastshield by slab masks. Thus, in principle the waste heat deposited in these elements either conducts to the central regions of the slab and is removed by radiation to the blastshield, or is cooled by a more direct approach. Both mechanisms were addressed in this study.

The optical path variation across the aperture is described as:

$$
O P L=\int n d z
$$

where $\boldsymbol{n}$ is the index of refraction, and the integration takes place along the beam propagation path, $z$. The index of refraction is a function of the temperature and stress, and can be represented as

$$
n=n_{o}+\frac{\partial n}{\partial T}\left(T-T_{o}\right)+\frac{\partial n}{\partial \sigma} \cdot \sigma
$$

where $\partial_{n} / \partial T$ is the scalar temperature coefficient, $\partial n / \partial \sigma$ is the stress-optic coefficient tensor, and $\sigma$ is the stress tensor. Thus, to properly determine the optical path variation in the slabs it is necessary to establish the temperature distribution, the stress distribution, and body deformations due to thermal expansion. 
Finite element calctlations on an isolated slab were performed using the TOPAZ3D ${ }^{1}$ and NIKE3D ${ }^{2}$ computer programs. Both use the finite element formulation, with TOPAZ3D used to evaluate the temperature distribution in the slab on the basis of the initial temperature distribution and boundary conditions. Incorporated in this calculation is radiation transport between the slab and blastshield. The heat transfer calculation is then followed by determination of the body deformation and stresses using the NIKE3D code and the temperature distribution from the TOPAZ3D calculation. The final step in the analysis is application of the calculated temperature, deformation, and stress fields in an optical propagation calculation to evaluate the optical path length variation across the aperture. ${ }^{3}$

Typical results are given in Figs. 2 and 3. In this calculation, the mechanical and optical properties of LG-770 were applied as summarized in Table 1. Of particular note is the negative $\mathrm{dn} / \mathrm{dT}$ of this laser glass. An important characteristic of this calculation is the masking of the edge claddings from radiative transport, so that the energy deposited in these regions had to conduct to the central regions of the slab for radiative dissipation to the blastshield. In Fig. 2, the temperature distribution across the slab aperture is given, showing in this instance that the edges are hotter than the central regions of the slab. During the entire recovery process, the peak temperature rise in the slab occurs in the corners where conductive dissipation of the edge cladding heat is more restrictive. Fig. 3 gives a decomposition of the optical path length changes, normalized by the maximum total optical path length variation. Fig. 3a, b, and $c$ show the isolated effects of temperature, displacement, and stress. It is noted that because of the negative $\partial_{n} / \partial T$, the colder central regions of the slab are phase delayed from the hotter outer regions. On the other hand, because the outer edges are hotter, thermal growth in these regions is greater resulting in a phase lag at the outer edged from the displacement, or growth effect. This clearly shows the potential for some cancellation of these two contributions. The overall optical path length variation is given in Fig. 3d, which primarily reflects the dominance of the greater thermal expansion contribution.

On the basis of these calculations, the edge-to-center optical path length variation was related to the edge-to-center temperature change as

$$
\triangle O P L_{e-c}=\gamma \cdot \Delta T_{e-c}
$$

where the subscript e-c denotes the edge-to-center difference, and $\gamma$ is the correlation constant. Correlating results from a number of calculations it was determined that

$$
\gamma=-0.45 \pm 0.1 \frac{\text { waves }}{\text { slab- } \text { pass }^{\circ} \mathrm{C}}
$$

For the NIF specification of 0.04 waves/slab/pass, this translates to a slab center-to-edge temperature limit of $0.09^{\circ} \mathrm{C}$.

The Beamlet thermal tests described in the introduction were used to validate this numerically determined temperature limit. Fig. 4 gives a plot of the horizontal temperature difference across the slab (center-to-edge) as a function of time. Also noted is a point in time where Hartman sensor data was used to determine that the $1 / 2$ cycle optical distortion in the probe beam was approximately 5 waves. This corresponds to an experimentally determined correlation coefficient, $\beta$, of

$$
\gamma=-0.57 \pm 0.05 \frac{\text { waves }}{\text { slab }- \text { pass }{ }^{\circ} \mathrm{C}}
$$

which is consistent with the numerically determined value. It is on this basis that confidence in a temperature difference limit of $0.09^{\circ} \mathrm{C}$ was established.

Having established the temperature difference limit across the aperture for thermal recovery, calculations were then used to quantify the effect of cooling options on thermal recovery. Two approaches were considered: (1) the NIF baseline concept of gas flow of $20 \mathrm{cfm}$ per lamp in the flashlamp cassettes through the entire cooling cycle, and (2) the addition of a mechanism to directly remove the edge cladding energy rather than rely on conducting that energy to the central regions of the slab for removal by radiative transport to the blastshields. One potential approach for cooling the edge cladding is depicted in Fig. 5. This approach consists of replacing the baseline flat edge cladding protective masks with a profiled mask that contains air cooling passages. Through inclusion of a direct conduction element, this affords a path for energy to be removed directly from the edge cladding. 
The performance of these cooling approaches is shown in Fig. 6 which gives plots of the center-to-edge temperature difference, and associated optical distortion difference, versus cooling time. The horizontal dashed lines denote the NIF recovery limit of $\pm 0.09^{\circ} \mathrm{C}$. The NIF baseline cooling approach of employing only active cooling of the flashlamps clearly has the potential to meet the NIF baseline 7 hour recovery requirement. On the other hand, clearly demonstrated is the need to employ edge cladding cooling in order to double the NIF shot rate, which would require 3 hours thermal recovery.

\section{GAS COLUMN OPTICAL DISTORTIONS}

Equally important to ensuring proper thermal recovery of NIF is consideration of the optical distortions in the gas columns within the amplifier cavities and beamtubes. Beamlet experiments were particularly important in establishing a thermal recovery criteria for this distortion contribution since precise numerical quantification of the optical distortions in this highly unstable convective flow was not an option in this study. Results of the Beamlet measurements are shown in Fig. 7, which gives the added half angle beam divergence as a function of a characteristic temperature difference. In this instance, the characterizing temperature quantity selected was the difference between the average temperature of the vertical surfaces in the cavity, and the top reflector. These represent hot and cold surfaces, in a buoyantly unstable configuration, which must be the case in any natural convection situation. They also represent the driving mechanisms for convective boundary layer flow on the slab faces, which was judged from near-field images to be the dominant mechanism in this Beamlet configuration. The shaded region denotes the experimental uncertainty of the beam divergence measurement. At a given temperature difference, the scatter in the added half angle data is related to the statistical variation in the alignment of convective disturbances. Based on the average of the statistical variation, this data indicates that a $2.5 \mu \mathrm{rad}$ added half angle distortion is associated with a $0.25^{\circ} \mathrm{C}$ characteristic temperature difference.

It must, however, be recognized that there are significant geometry differences between the Beamlet and NIF amplifier architectures. In particular, the NIF system consist of a 4-slab high stack as opposed to the 2-slab high Beamlet configuration. Thus, the height of gas columns in the cavity region and beamtubes will be twice that of Beamlet. Preliminary estimates of this effect, based on linear stability theory and boundary layer growth theory ${ }^{5}$ suggest as much as a 2 times greater sensitivity to temperature differences in NIF than in Beamlet. Thus, it is reasonable to reduce the NIF acceptable temperature limit to $0.13^{\circ} \mathrm{C}$, half of the value experimentally determined for Beamlet. It must be emphasized that this is only a preliminary projection, and awaits further analysis and experiments to precisely quantify the NIF thermal recovery conditions as they relate to gas column convective disturbances.

Fig. 8 presents results of the effect of several thermal recovery cooling conditions on the average slab temperature rise above ambient temperature. These results were obtained using the lumped mass thermal model, with validation calculations performed using the two- and three-dimensional finite-element models. In the case of actively cooled flashlamps, the average temperature rise in the slab is indicative of two key convective flow driving temperature differences: (1) the difference between the slab and blastshield, since at later times in the recovery cycle the blastshield will be at ambient temperature, and (2) the difference between the slab and beamtube structure temperature since the beamtubes are subject to ambient. temperature conditions.

Consider first the curves for ambient gas flow at $20 \mathrm{cfm}$ and $5 \mathrm{cfm}$ per flashlamp. Clearly indicated is the potential for the NIF baseline to meet the 7 hour recovery requirements associated with the projected NIF temperature difference limit of $0.13^{\circ} \mathrm{C}$. Equally evident is the inability of this cooling scenario to provide any reasonable cooling margin for the 7 hour recovery case, as well as achieve recovery in 3 hours. Equally evident is the necessity for the $20 \mathrm{cfm}$ flow rate, as evidenced by the striking inability of a $5 \mathrm{~cm}$ per lamp flowrate to even remotely meet NIF requirements. Also note the rise in the slab temperature during the very early states of thermal recovery. This is a result of radiative heat transfer from the hot flashlamps that persists until the cooling gas has reduced the flashlamp temperature to a value below that of the slabs.

The final curve in Fig. 8 is for a $5 \mathrm{cfm}$ per lamp flowrate, but with a temperature modified recovery cycle as indicated in Fig. 9. In this case, at the start of the recovery cycle the flashlamp cooling gas is chilled to $2^{\circ} \mathrm{C}$ below ambient. This is maintained until 2.6 hours into the recovery cycle when the gas is returned to ambient temperature for an additional 0.4 to 1.4 hours. The purpose of initially chilling the gas is to provide a stronger driving temperature difference for radiative transport. Since the blastshields are in contact with this cooling flow, after roughly 0.5 hours they will be nearly in thermal equilibrium 
with the cooling gas. This added temperature difference between the cooler blastshields and the hotter laser slabs dramatically enhances radiative transport. However, after 2.6 hours, while the slabs have been effectively reduced to ambient temperature, the blastshields and flashlamps are at a temperature $2^{\circ} \mathrm{C}$ below ambient. Without further action, this would result in a hydrodynamically unstable condition. In order to rectify this situation, the cooling flow is returned to ambient temperature for the time necessary to re-warm the blastshields and flashlamps to ambient temperature. This chilled gas cooling scenario has two significant effects on NIF thermal recovery: (1) recovery in 3 hours or less is clearly possible, and (2) accelerated recovery can potentially be achieved with a significantly lower gas flow rate, which will have a beneficial impact on the cost of the NIF cooling system through a requirement for smaller ducts and fans.

Since the optical distortions in the gas columns are dependent on the optical properties of the gas itself, it is useful to consider the consequences of filling the amplifier cavities and beamtubes with a gas other than air or nitrogen. Of particular interest is helium. To first order, for incoherent disturbances such as those present in the gas columns, the change in optical path length is approximated as

$$
\Delta O P L=\delta \sqrt{\frac{L}{\delta}} \beta \frac{\Delta T}{T_{o}}
$$

where $\delta$ is an ensemble characteristic disturbance scale size in the gas columns, $\mathrm{L}$ is the total propagation length through gas in the amplifier cavities and beamtubes, $\Delta T$ is a characteristic temperature perturbation in the gas, and $T_{0}$ is the bulk gas temperature. The quantity $\beta$ is the Gladstone-Dale constant for the fill gas. ${ }^{6}$ Noting that that the Gladstone-Dale constant for helium is $1 / 8$ that of nitrogen, we project an allowable temperature difference in a helium filled system to be 8 times that of a nitrogen filled system. The potential NIF temperature difference limit for a helium filled system is shown in Fig. 8. This clearly shows the leverage afforded by a helium fill since adequate recovery can potentially be achieved with ambient gas flow rates of a little as $5 \mathrm{cfm}$ per flashlamp.

\section{SUMMARY AND CONCLUSIONS}

In summary we note that preliminary calculations have been performed to predict NIF thermal recovery for various cooling approaches. These calculations of thermal transport in the amplifier cavity range in complexity from a lumped-mass thermal approximation to detailed three-dimensional finite element simulations. In addition, experiments were performed on the Beamlet facility where temperature differences in the slabs as well as between the slab and surrounding surfaces were measured. These measured temperature differences were correlated to simultaneously measured optical distortions. This combined modeling and experimental approach has allowed us to draw the following conclusions:

1. The NIF slab optical distortion limit of 0.04 waves/slab/pass is related to a temperature difference across the aperture of no more than $0.09^{\circ} \mathrm{C}$.

2. The NIF gas column optical distortion limit of $2.5 \mu \mathrm{rad}$ additional half-angle beam divergence, due to convective effects, is related to a projected surface temperature difference in the NIF geometry amplifier cavity of $0.13^{\circ} \mathrm{C}$.

3. The NIF baseline cooling approach consisting of a $20 \mathrm{cfm}$ per flashlamp ambient temperature flow in both the central and side flashlamp cassettes appears adequate to meet the NIF recovery criteria after 7 hours of thermal recovery.

4. Doubling the NIF shot rate, which means reducing the thermal recovery time to 3 hours, requires consideration of advanced cooling options such as chilling the flashlamp cooling gas and direct cooling of the slab edge claddings.

5. Filling the amplifier cavities and beamtubes with helium, which is much less optically sensitive to temperature perturbations, dramatically relaxes the NIF temperature recovery criteria associated with convective gas currents. This allows more rapid recovery to be achieve with lower flow rates of ambient gas than can be achieved with a nitrogen filled system.

It must be emphasized that these results and conclusions are preliminary. To refine our understanding, more detailed two- and three-dimensional thermal models are under development, and thermal tests similar to those conducted in the Beamlet facility are planned for the AMPLAB facility which is a mock-up of the NIF amplifier geometry. The AMPLAB tests will be used to experimentally quantify the recovery conditions for NIF. This combination of refined analysis and AMPLAB 
thermal tests will allow to to precisely specify NIF recovery requirements and verify that the NIF baseline approach of $20 \mathrm{cfm}$ per flashlamp cooling in the side and central cassettes is adequate to meet NIF baseline cooling needs.

\section{ACKNOWLEDGMENTS}

This work was performed under the auspices of the U.S. Department of Energy by the Lawrence Livermore National Laboratory under contract no. W-7405-Eng-48. The authors wish to thank Al Erlandson, Mark Rotter, Buzz Pedrotti, and Rick Sawicki for helpful discussion throughout the course of this effort.

\section{REFERENCES}

1. A. B. Shapiro, "TOPAZ3D - A three-dimensional finite element heat transfer code," LINL, UCID-2-848, August 1985.

2. B. N. Maker, et. al., "NIKE3D - A Nonlinear, Implicit, Three-dimensional Finite Element Code for Solid and Structural Mechanics," LLNL, UCRL-MA-105268 Rev. 1, April 1995.

3. The optics code applied in this analysis is an adaptation of: S. K. Doss, "Physics and Mathematics of the BREW Code," 1986 Laser Program Annual Report, LLNL, UCRL-50021-86, pp. 7-132 - 7-135, November 1987.

4. For a discussion of the application of linear stability theory to the amplification of disturbances in natural convective flows see for example: B. Gebhart, Heat Transfer, McGraw-Hill, 1971.

5. For a discussion of natural convection boundary layer grow see for example: F. M. White, Viscous Fluid Flow, McGraw-Hill, New York, 1974.

6. R. A. Hass, "Propagation of Laser Energy," 1977 Laser Program Annual Report, LLNL, UCRL-50021-77, pp. 7$16-7-20$.

Table 1

Thermo-Mechanical and Optical Properties of LG-770 (Schott Glass Technologies)

\begin{tabular}{|l|c|c|}
\hline \multicolumn{1}{|c|}{ Property } & Unit & Value \\
\hline Thermal conductivity & $\mathrm{W} / \mathrm{m}-\mathrm{K}$ & 0.52 \\
\hline Density & $\mathrm{kg} / \mathrm{m}^{3}$ & 2830 \\
\hline Specific heat & $\mathrm{kJ} / \mathrm{kg}-\mathrm{K}$ & 0.72 \\
\hline Elastic modulus & $\mathrm{GPa}$ & 50.1 \\
\hline Poisson's ratio & & 0.256 \\
\hline Thermal expansion coefficient & $\mathrm{K}^{-1}$ & $11 \times 10^{-6}$ \\
\hline Index of refraction & & 1.516 \\
\hline dn/dT & $\mathrm{K}^{-1}$ & $-5.4 \times 10^{-6}$ \\
\hline $\begin{array}{l}\text { Piezo-Optic coefficients } \\
\pi_{11}\end{array}$ & $\mathrm{TPa}^{-1}$ & 1.526 \\
$\pi_{12}$ & $\mathrm{TPa}^{-1}$ & 2.54 \\
\hline
\end{tabular}




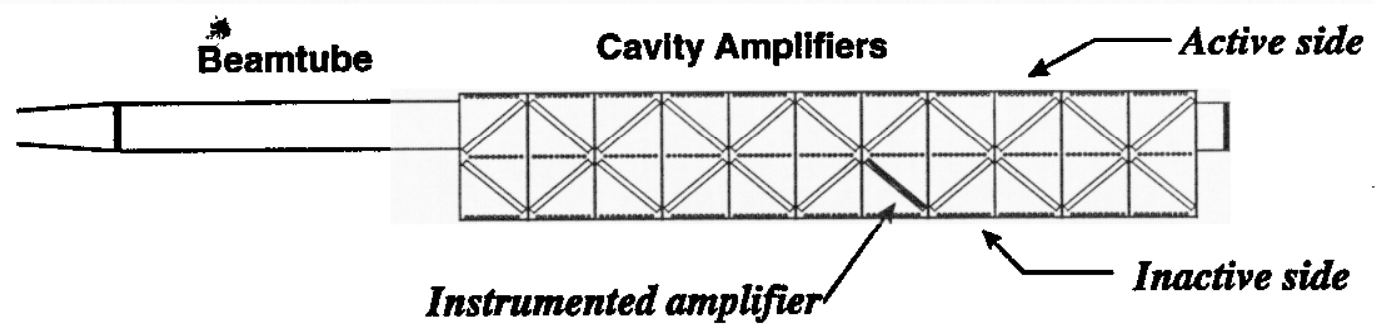

(a)

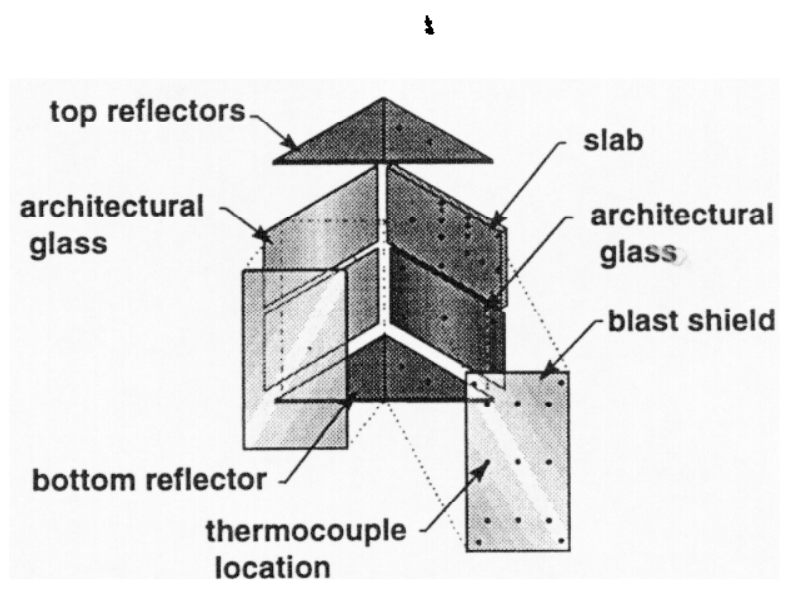

(b)

Fig. 1 - Location of instrumented amplifier on the inactive side of Beamlet, and the location of thermocouples on the slab, blastshield and reflectors. (a) a plan view of the cavity amplifier and the instrumented amplifier location. (b) an exploded view of the instrumented amplifier showing thermocouples on the slab, lower architectural glass panel, blastshield, and top and bottom reflectors.

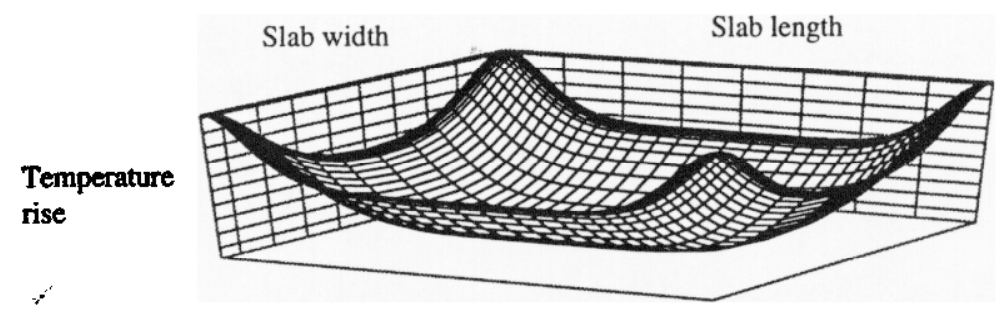

Fig. 2 - The calculated temperature distribution across the slab aperture. This calculation shows hotter temperatures toward the slab edges, with the largest temperature rise occurring in the slab corners. In this figure, the maximum and minimum temperature rises are $0.66^{\circ} \mathrm{C}$ and $0.17^{\circ} \mathrm{C}$ respectively. 


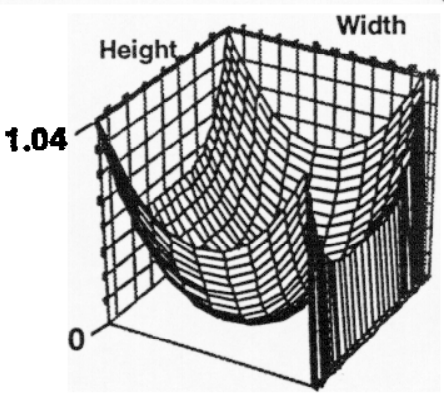

(a)

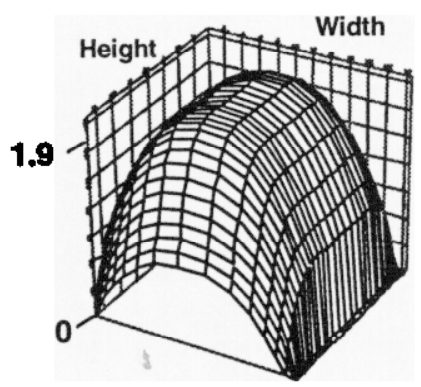

(c)

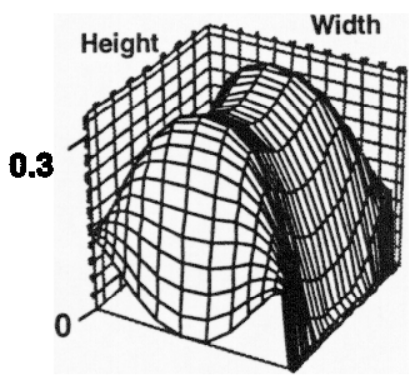

(b)

(d)

Fig. 3 - Calculated contributions to the optical distortion in the laser slabs, normalized by the maximum total optical distortion. (a) the $\mathrm{dn} / \mathrm{dT}$ contribution. (b) the displacement (thermal expansion) contribution. (c) the thermal stress contribution. (d) the total of all contributions.

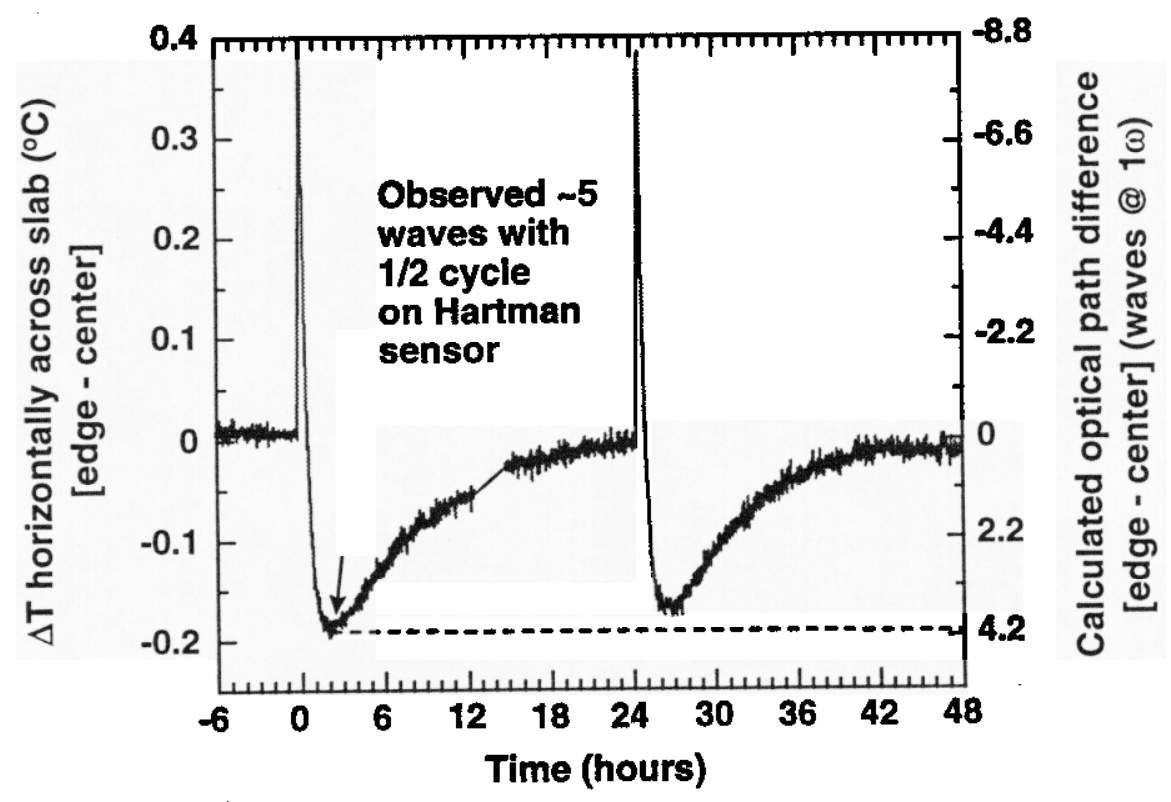

Fig. 4 - Experimentally measured values of the center-to-edge temperature difference, and corresponding calculated optical path difference. The single point experimentally measured distortion is indicated, denoting the good agreement between experiments and model results. 


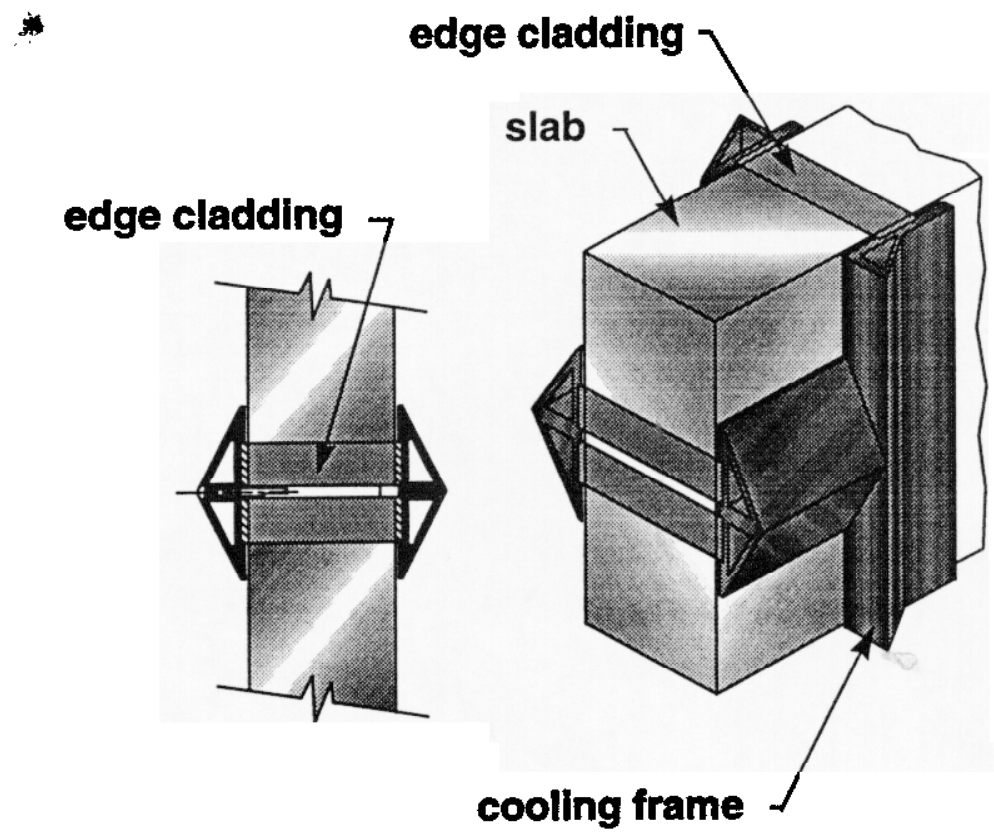

Fig. 5 - A potential edge cladding cooling approach consisting of gas cooled masks. The purpose of cooling the edge cladding is to remove the edge cladding thermal load before it can conduct into the slab and enhance the temperature gradients across the aperture.

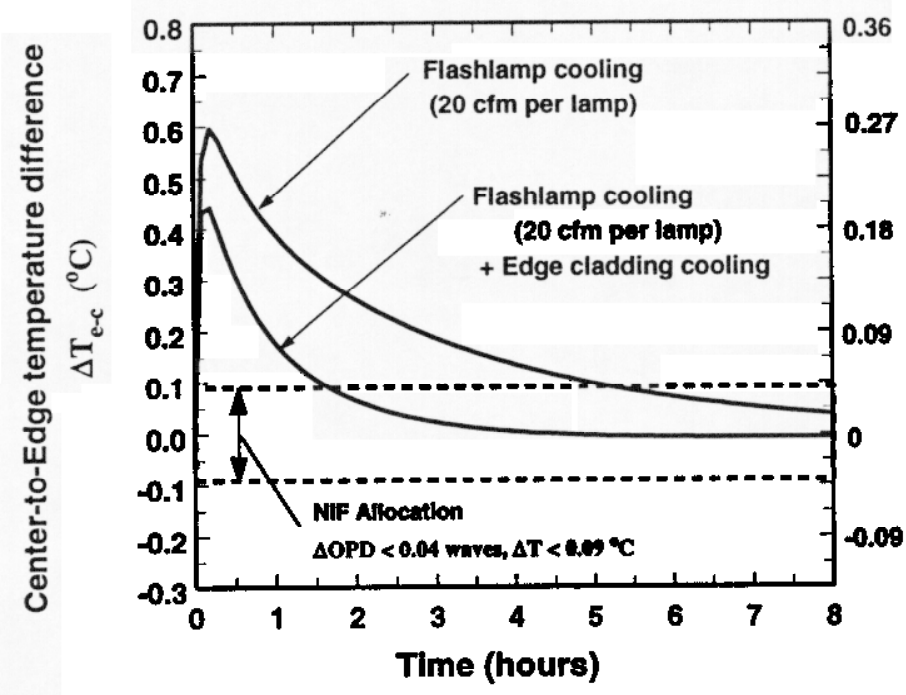

Fig. 6 . The calculated effect of flashlamp and edge cladding cooling on the reduction of temperature gradients and optical distortions across the aperture. These calculations indicate that flashlamp cooling is likely adequate for an 8 hour shot period, but to achieve a 4 hour shot period would require edge cladding cooling. 


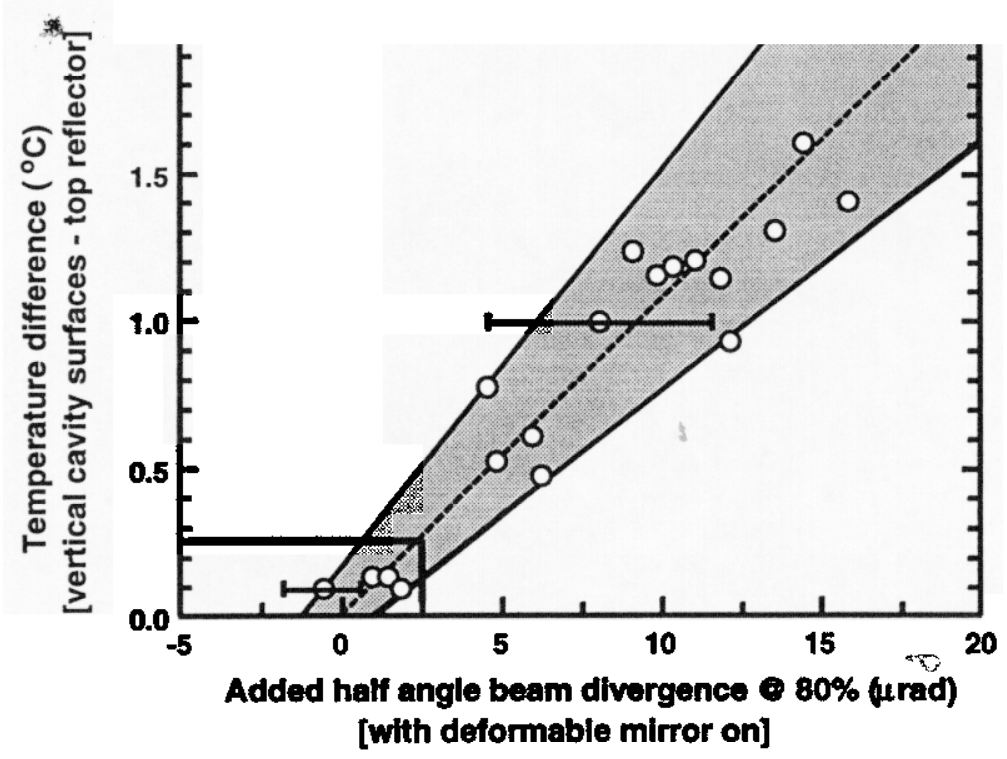

Fig. 7 - The correlation of added half angle beam divergence with the characteristic temperature difference in the Beamlet experiments. Based on the average added divergence, a $2.5 \mu \mathrm{rad}$ added beam divergence corresponds to $0.25^{\circ} \mathrm{C}$ temperature difference in Beamlet.

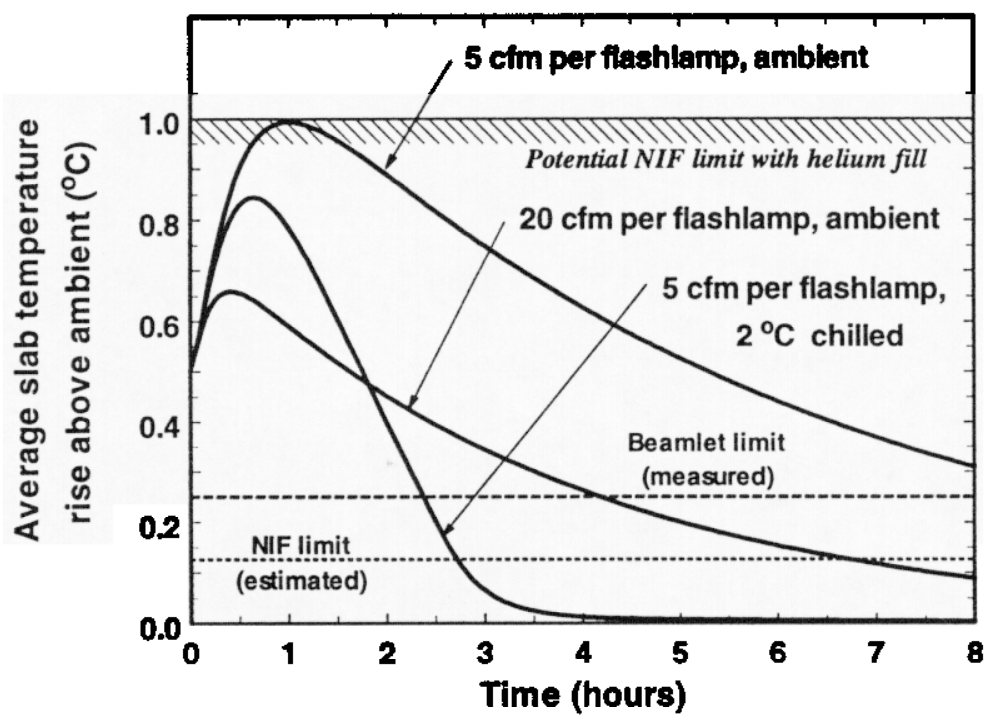

Fig. 8 - The effect of flashlamp cooling gas flow rate and temperature on thermal recovery of the laser slabs. In this plot, the average temperature rise is representative at the latter stages of recovery of the convective flow driving temperature differences in the amplifier cavity and beamtubes. These calculations show that the NIF $20 \mathrm{cfm}$ per lamp cooling flow is likely adequate for an $\mathbf{8}$ hour shot period, but chilling the gas or the use of helium filled amplifier cavities and beamtubes would be required to achieve a 4 hour shot period. 
$\Rightarrow$

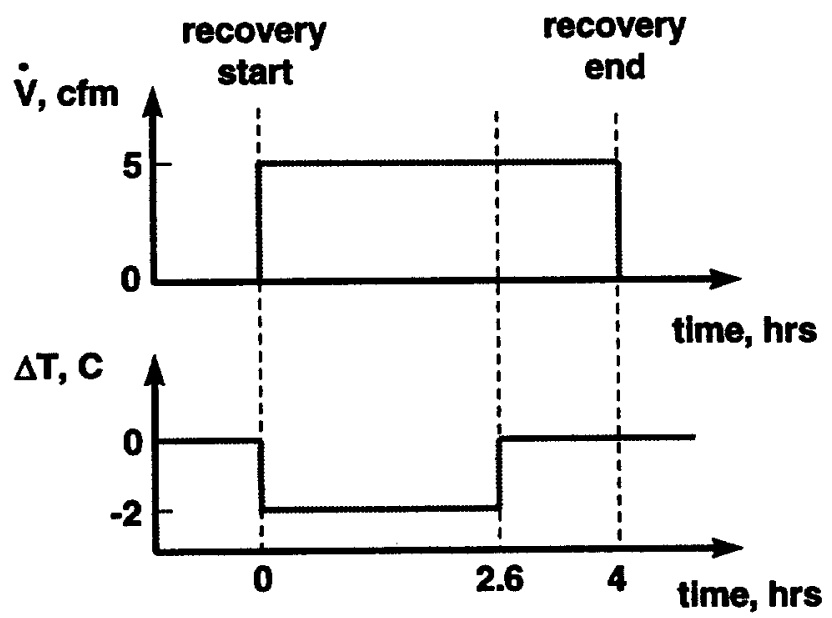

Fig. 9 - The volume flow rate and temperature profiles used in the chilled gas recovery scenario of Fig. 8. Note that at 2.6 hours after the start of recovery, the gas is rewarmed to ambient temperature. 


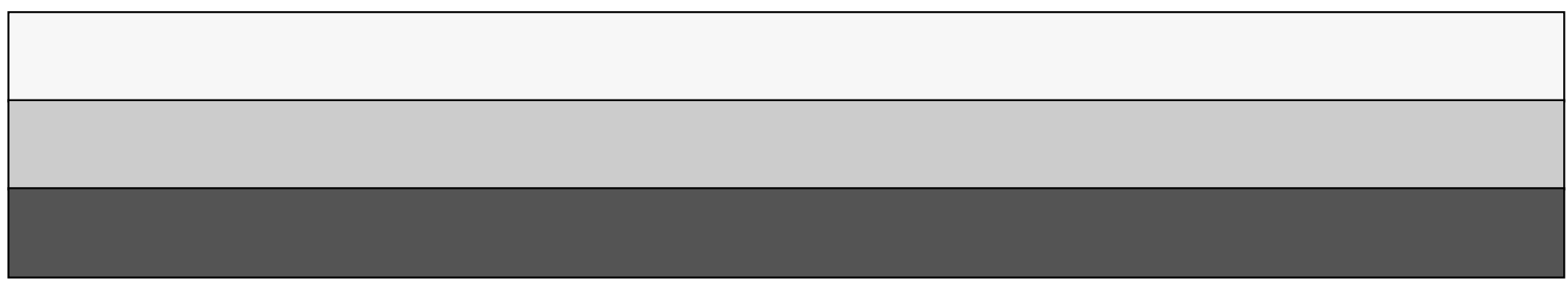

